\section{Smoking cessation: what the health service can do}

\author{
Effectiveness matters Volume 3, Issue 1, March 1998
}

Published by NHS Centre For Reviews And Dissemination, The University Of York

\section{Summary}

- In 1995 tobacco was responsible for 20,000 deaths of people over 35 years of age in the UK and rates of tobacco use are increasing, particularly in young people.

- Research evidence shows that there are highly cost-effective ways to help people stop smoking.

- Health professional advice about quitting can achieve cessation rates of $2 \%$. This quit rate can be increased to around $12 \%$ in motivated patients by adding nicotine replacement therapy.

- Health professionals should systematically identify patients who smoke and encourage and support them to stop.

- The use of nicotine replacement therapy should be encouraged in those smokers who are motivated to quit.

- Health professionals should encourage and help pregnant women to stop smoking.

- Some health professionals would benefit from training in smoking cessation techniques.

- Health authorities and other health service commissioners should develop co-ordinated smoking cessation strategies and fund their implementation.

The review identifies the evidence relating to the effectiveness of various types of interventions.

\section{Interventions with good evidence of effectiveness}

- Brief advice from a health professional

Brief advice to stop smoking given by health professionals, taking around 3 minutes, has been shown to decrease the proportion of people smoking by around $2 \%$ when compared with patients who did not receive any advice). Given the large number of smokers who have contact with health professionals, a $2 \%$ reduction represents a very significant population health gain. Increasing the intensity of advice (i.e. the time spent giving advice on smoking and the duration of followup) improves the effectiveness, decreases the proportion smoking by around 3-5\%. However, more intensive advice may not always be feasible in primary care because of the resources required.

- Nicotine replacement therapy with advice

Trials have shown that the use of nicotine replacement therapy
(NRT) decreased the proportion of people smoking by around $12 \%$ in more motivated patients who were selfreferred. In all these studies some form of additional support, ranging from brief advice to more intensive forms of counselling, was provided alongside the NRT.

- Advice \& support to pregnant women

The provision of advice to pregnant women has also been shown to be effective.

\section{Interventions with insufficient evidence of effectiveness}

- use of antidepressants

- Aversive conditioning

- Acupuncture

- Hypnosis

- Meca mylamine

- Self help materials: booklets, pamphlets, manuals

\section{Cost effectiveness}

Analyses have consistently reported that smoking cessation interventions are relatively cheap ways of saving lives and reducing morbidity. Therefore the interventions reported above are a good use of NHS time and resources.

\section{Encouraging smoking cessation}

There is evidence that the NHS could do more to promote smoking cessation. In a recent national survey, for example, only $38 \%$ of current smokers recalled being given advice on smoking by their GP and 13\% by someone else at the surgery. There is good evidence that training health professionals to offer smoking cessation interventions in primary care settings and providing chart or computer-based reminders increases the frequency with which such interventions are offered.

\section{Recommendations}

Smoking cessation interventions are highly cost effective therefore:

- Health professionals should identify smokers, encourage and support them to stop and provide follow up.

- They should encourage the use of nicotine replacement therapy in those smokers who are motivated to quit

- Pregnant women should be offered intensive advice and support to stop smoking. Health authorities and other commissioners of health care should develop local strategies for promoting smoking cessation which build on national initiatives such as 'No Smoking Day' and 'Quitline'(0800 002200). 